\title{
Análise dos programas ambientais de saúde no licenciamento ambiental de hidrelétricas na Amazônia legal brasileira
}

\author{
Analysis of environmental health programs on environmental hydroelectric licensing in \\ brazilian legal Amazon
}

\begin{abstract}
Análisis de los programas de salud ambiental en licencias hidroeléctricas ambientales en el Amazón legal brasileño
\end{abstract}

Regiane Cristina Neto Okochi ${ }^{1}, 2^{\star}$, Elineide Eugênio Marques².

\begin{abstract}
RESUMO
Objetivo: Analisar de forma crítica no âmbito dos processos de licenciamentos ambientais em hidrelétricas no Brasil, referentes aos programas ambientais de saúde direcionados para as populações afetadas nos reservatórios hidrelétricos de Belo Monte, Estreito e Santo Antônio, localizados na Amazônia Legal brasileira. Métodos: Pesquisa documental descritiva qualitativa em 3 áreas na Amazônia Legal brasileira, duas na região norte; UHE Belo Monte, localizada no rio Xingu e UHE Santo Antônio, no rio Madeira e uma área na região nordeste do Brasil, UHE Estreito, no rio Tocantins. Público estimado em 772.061. Instrumento de pesquisa elaborado pelo método Delphi. Resultados: Das 3 áreas de estudo, 107 programas ambientais, apresentaram se descritos no estudo de impacto ambiental (EIA), dentre os quais (10) programas e projetos referentes a saúde da população afetada. A estruturação e sistematização, apresentam incipiência no padrão técnico científico, referente a temática do estudo e ressaltam apenas uma doença como potencial de risco, a Malária. Conclusão: Evidencia-se a necessidade de um balanço crítico do modelo que vem sendo usado para a construção dos programas ambientais de saúde. Medidas técnicas, científicas e epidemiológicas efetivas devem ser introduzidas, além das considerações ambientais no processo decisório do licenciamento ambiental.
\end{abstract}

Palavras-chave: Hidrelétricas, Licenciamento, Programas ambientais, Saúde da população.

\begin{abstract}
Objective: To critically analyze the environmental licensing processes in hydroelectric dams in Brazil, regarding the environmental health programs directed to the affected populations in the Belo Monte, Estreito and Santo Antônio hydroelectric reservoirs, located in the Brazilian Legal Amazon. Methods: Qualitative descriptive documentary research in 3 areas in the Brazilian Legal Amazon, two in the northern region; Belo Monte HPP, located on the Xingu River and Santo Antônio HPP, on the Madeira River and an area in northeastern Brazil, Estreito HPP, on the Tocantins River. Estimated audience at 772,061. Research instrument elaborated by the Delphi method. Results: Of the 3 areas of study, 107 environmental programs were described in the Environmental Impact Study (EIA), including (10) programs and projects concerning the health of the affected population. The structuring and systematization, are incipient in the scientific technical standard, referring to the study theme and highlight only one disease as a potential risk, Malaria. Conclusion: There is a need for a critical balance of the model that has been used for the construction of environmental health programs. Effective technical, scientific and epidemiological measures should be introduced, in addition to environmental considerations in the environmental licensing decision-making process.
\end{abstract}

Key words: Hydropower, Licensing, Environmental Programs, Population Health.

1Instituto Federal do Tocantins (IFTO), Gurupi - TO. *E-mail: regianeokochi@gmail.com

2 Programa de Pós-graduação em Ciências do Ambiente. Universidade Federal do Tocantins (UFT), Palmas - TO. 


\section{RESUMEN}

Objetivo: Analizar críticamente los procesos de concesión de licencias ambientales en las presas hidroeléctricas en Brasil, en relación con los programas de salud ambiental dirigidos a las poblaciones afectadas en los reservorios hidroeléctricos de Belo Monte, Estreito y Santo Antônio, ubicados en la Amazonia Legal de Brasil. Métodos: Investigación documental descriptiva cualitativa en 3 áreas en la Amazonia Legal Brasileña, dos en la región norte; Belo Monte HPP, ubicado en el río Xingu y Santo Antônio HPP, en el río Madeira y un área en el noreste de Brasil, Estreito HPP, en el río Tocantins. Audiencia estimada en 772.061. Instrumento de investigación elaborado por el método Delphi. Resultados: De las 3 áreas de estudio, 107 programas ambientales se describieron en el Estudio de Impacto Ambiental (EIA), incluidos (10) programas y proyectos relacionados con la salud de la población afectada. La estructuración y la sistematización son incipientes en el estándar científico-técnico, refiriéndose al tema del estudio y destacan solo una enfermedad como riesgo potencial, la malaria. Conclusión: Existe la necesidad de un equilibrio crítico del modelo que se ha utilizado para la construcción de programas de salud ambiental. Se deben introducir medidas técnicas, científicas y epidemiológicas efectivas, además de las consideraciones ambientales en el proceso de toma de decisiones sobre licencias ambientales.

Palabras clave: Hidroelectricidad, Licencias, Programas Ambientales, Salud de la Población.

\section{INTRODUÇÃO}

Programas ambientais no contexto do licenciamento ambiental em hidrelétricas brasileiras fundamentam se na política nacional ambiental. Dentre eles o programa ambiental de saúde para a população afetada. Esses programas são caracterizados por um conjunto de planos, identificados a partir da elaboração dos estudos ambientais, com cronograma executivo, plano de trabalho operacional e definição das ações a serem desenvolvidas nas etapas de implantação e operação da atividade e ainda monitoramento de indicadores ambientais (BRASIL, 2015).

No entanto os estudos e as avaliações de impactos ambientais realizados não contemplam a saúde de forma sistemática, integral e harmonizada com os princípios da promoção da saúde e da sustentabilidade socioambiental e divide o público-alvo em população afetada e trabalhadores da obra. Sua concepção perpassa por diversas áreas do conhecimento ambiental, como o meio físico, biótico e socioeconômico (BARBOSA EM, BARATA MM, HACON SD, 2012).

Existem ainda limitações e dificuldades institucionais de gestão, infraestrutura e de natureza técnica para o estabelecimento de um processo integrado de vigilância e controle dos aspectos do ambiente e saúde nesse contexto (SILVA JM, et al. 2009).

O contexto das usinas hidrelétricas (UHE) é marcado por contradições entre efeitos positivos apresentados como um legado à população afetada, além da geração temporária de emprego e renda e efeitos negativos, que, com o afluxo populacional tanto de trabalhadores como de pessoas atraídas pela obra, ocasionam modificações intensas no território, representando pressão sobre os serviços públicos locais, principalmente para o sistema de saúde, que, despreparado para aguentar o aumento de serviços, pleiteia a expansão dos recursos humanos e financeiros (SILVEIRA M,2016).

Apesar das contradições, estudos de planejamento energético do Brasil, indicam a fonte hidráulica como importante para a expansão futura do setor elétrico nacional (BRASIL, 2018).

Partindo do pressuposto da expansão do setor elétrico, que culminara na construção de novos projetos hidrelétricos na Amazônia legal brasileira e que esses projetos são aprovados via licenciamento ambiental e junto a eles virão os programas ambientais de saúde. Unindo a esse contexto a diversidade elevada dos ambientes tropicais da região assim se justificou esse estudo. Além disso teve como objetivo uma análise crítica e comparativa nos processos de licenciamentos ambientais referentes aos programas ambientais de saúde direcionado para as populações afetadas nos reservatórios hidrelétricos de Belo Monte, Estreito e Santo Antônio. 


\section{MÉTODOS}

Optou-se pela pesquisa documental comparativa ancorada na abordagem qualitativa, por meio de análise documental do conteúdo disponível em fonte de papel e multimídia eletrônica, utilizando o método Delphi, uma Ferramenta metodológica aplicável em situações decisórias e diagnósticas conhecido também como Técnica Delphi ou Método Delfos, esse método de pesquisa tem amplo uso nas áreas das ciências da saúde e ciências humanas (ANTUNES MM, 2014). Através do estudo de três usinas hidrelétricas brasileiras com perspectiva interpretativa. Para a realização da análise documental foi criado um instrumento para categorização analítica dos documentos. Para uso desse método de análise foram consideradas as contribuições de Kuenzer (1998) que refere que as categorias servem de critério de seleção e organização das teorias e dos fatos a serem investigados, a partir, da finalidade da pesquisa, fornecendo-lhe o princípio de sistematização que vai Ihe conferir sentido, cientificidade, rigor, importância e também Minayo MCS (2004) que esclarece que os conceitos mais importantes dentro de uma teoria são as categorias.

Para a análise documental das categorias, foram utilizados os bancos de dados do Instituto Brasileiro de Meio Ambiente (IBAMA). E os documentos técnicos pesquisados nos processos de licenciamento ambiental foram: Estudo de Impacto ambiental (EIA) Termos de Referência e Programas Ambientais de saúde da área de estudo. Adotou se como técnica da pesquisa a análise de conteúdo, a análise de conteúdo diz respeito às técnicas de pesquisa que permitem tornar replicáveis e válidas inferências sobre dados de um determinado contexto, por meio de procedimentos especializados e científicos (CANCIO JA, 2008).

\section{Caracterização da área de estudo}

Duas áreas na região norte; Usina Hidrelétrica (UHE) Belo Monte, localizada no rio Xingu e UHE Santo Antônio, no rio Madeira e uma área na região nordeste do Brasil, UHE Estreito, no rio Tocantins. As três áreas estão localizadas na Amazônia Legal brasileira (Figura 1).

Figura 1- Localização das áreas de estudo e os municípios com a área de influência direta.

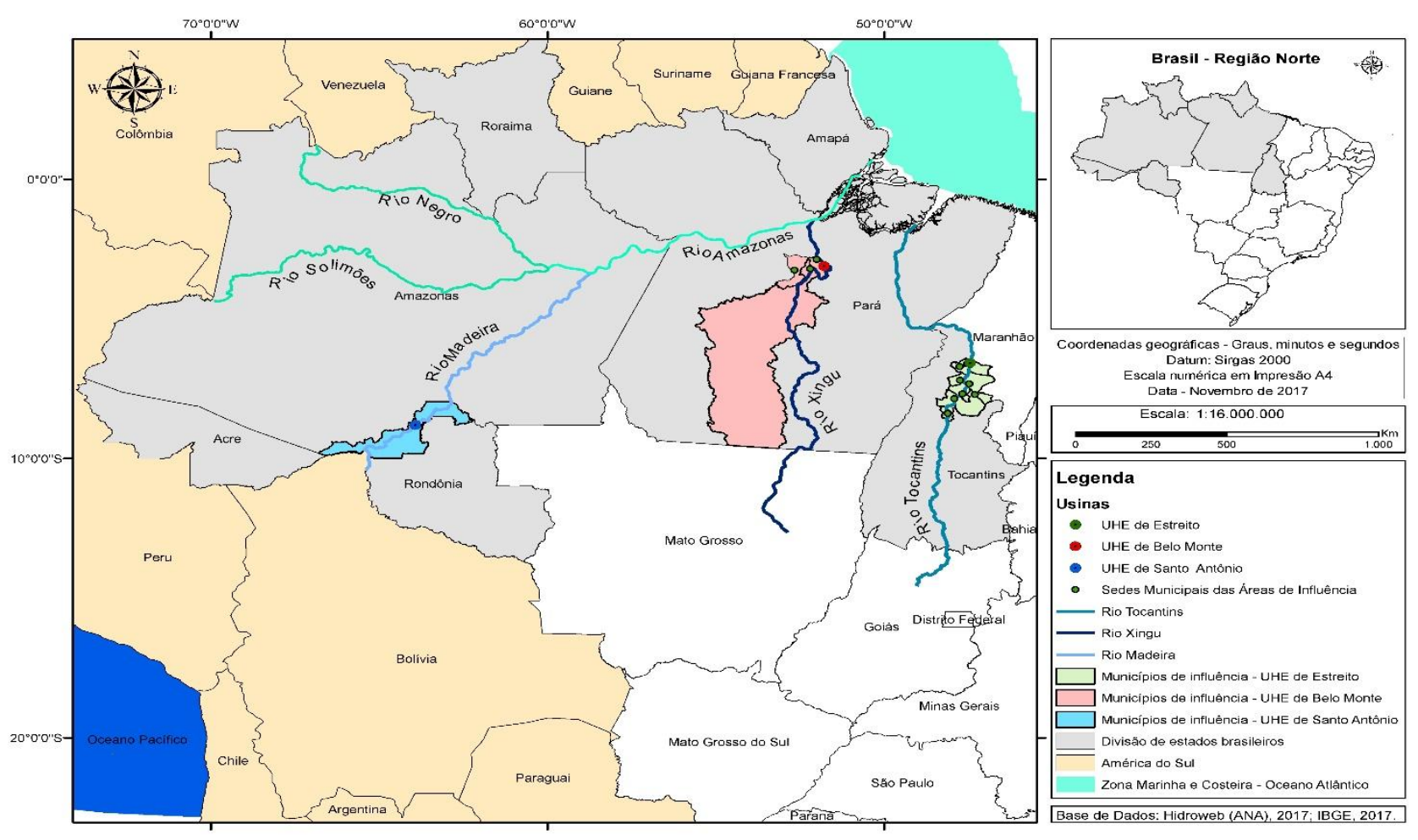

Fonte: HidroWeb da Agência Nacional de Águas (ANA) e Base cartográfica do IBGE, consultados em 2017. Elaborado por Ribeiro e Okochi (2019). 
O critério de inclusão das áreas de estudo se deu por ordem de instalação cronológica recente, com menos de uma década em operação e geração de energia elétrica, sob o mesmo regime jurídico, instalados na Amazônia Legal brasileira. Excluíram se demais projetos que não se encaixaram nos critérios de inclusão.

Para fins metodológicos foram denominadas de área 1, 2 e 3, em ordem cronológica decrescente, considerando o período inicial de implantação dos projetos, apontado no (Quadro 1).

Quadro 1 - Definição cronológica decrescente das áreas de estudo, período de 2007 a 2011.

\begin{tabular}{|l|l|l|}
\hline Área de estudo & Período inicial de implantação & UHE \\
\hline Área 1 & 2011 & Belo Monte \\
\hline Área 2 & 2008 & Santo Antônio \\
\hline Área 3 & 2007 & Estreito \\
\hline
\end{tabular}

Fonte: Autores (2019).

A área de estudo 1, compreende toda área de Influência direta (AID) referente a UHE de Belo Monte, e se localiza na área denominada de Volta Grande do rio Xingu, entre os paralelos $3^{\circ} 00^{\prime}$ e $3^{\circ} 40^{\prime} \mathrm{S}$ e os meridianos $51^{\circ} 30^{\prime}$ e $52^{\circ} 30^{\prime} \mathrm{W}$ e o eixo do barramento nas coordenadas $03^{\circ} 07^{\prime} 35^{\prime \prime} \mathrm{S}$ e $51^{\circ} 46^{\prime} 30^{\prime \prime} \mathrm{W}$ (EIA, 2009). A AID da área 1, de acordo com o EIA (2009), abrange os municípios de Altamira, Brasil Novo e Vitória do Xingu, no estado do Pará. E apresenta uma população estimada de 137.773 pessoas em 2015 (IBGE, 2016).

A área de estudo 2, compreende toda AID referente a UHE Santo Antônio, situada nas coordenadas $8^{\circ}$

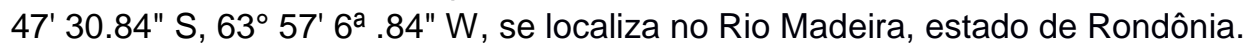

A AID da área 2, de acordo com o EIA (2005), abrange diretamente o município de Porto Velho, no estado de Rondônia e apresenta uma população estimada de 502.748 pessoas em 2015 (IBGE, 2016).

A área de estudo 3, compreende toda AID referente a UHE Estreito, situada nas coordenadas geográficas aproximadas de $06^{\circ} 35^{\prime} 11^{\prime \prime} \mathrm{S}$ Latitude, e $47^{\circ} 27^{\prime} 27^{\prime \prime}$ W Longitude (EIA, 2006), localizada no Rio Tocantins (EIA, 2006).

A AID da área 3, abrange diretamente doze municípios, sendo dois municípios na região nordeste, Carolina e Estreito, no estado do Maranhão, e dez municípios na região norte do Brasil, Aguiarnópolis, Babaçulândia, Barra do Ouro, Darcinópolis, Filadélfia, Itapiratins, Palmeirante, Goiatins, Palmeiras do Tocantins e Tupiratins, no estado do Tocantins (BRASIL, 2007). Apresenta uma população estimada de 118.727 pessoas em 2015 (IBGE, 2016).

A área de estudo 1 é caracterizada pelo Rio Xingu que pertence ao grupo dos rios de águas claras, pobres em sedimentos, mas em outras áreas podem ser ricas em matéria dissolvida (AYRES JM, 1995).

O Rio Xingu possui uma extensão de $1.500 \mathrm{~km}$, desde suas nascentes no Planalto Central Brasileiro até sua foz no rio Amazonas (MIRANDA EE, et al. 1988). Nasce a oeste da Serra do Roncador e ao norte da Serra Azul, no leste do Mato Grosso. Corre na direção sul-norte, paralelo aos rios Tapajós e Tocantins e após percorrer pouco mais de 2.000 km, deságua ao sul da ilha de Gurupá (Pará), na margem direita do Amazonas, do qual é um dos maiores afluentes (SALOMÃO RP, et al. 2007).

A área de estudo 2 é caracterizada pelo Rio Madeira, afluente do rio Amazonas apresenta aproximadamente $1.450 \mathrm{~km}$ de extensão, corta a porção sudoeste e noroeste dos estados do Amazonas e Rondônia, nasce na Bolívia, a partir da confluência dos rios Guaporé, Mamoré, Beni e Madre de Dios e é identificado como de água branca devido à grande carga de sedimentos em suspensão (HORBE AM, 2013).

A área de estudo 3 é caracterizada pelo Rio Tocantins que flui de Sul para Norte, desde sua cabeceira no Planalto Central brasileiro até a foz no rio Pará, é formado pelos rios das Almas e Maranhão, e com extensão total de $1960 \mathrm{~km}$ até sua foz no Oceano Atlântico (NERES JC, 2008). Tem o rio Araguaia como seu principal afluente, na margem direita, onde destacam-se os rios Bagagem, Tocantizinho, Paranã, Rio do Sono, Manoel Alves e Farinha, e na margem esquerda, o rio Santa Teresa e Itacaúnas (ANA, 2006). 
A dimensão populacional total das três áreas de estudo, apresentam uma estimativa de 772.061 indivíduos, subdivididos em 16 municípios brasileiros, nos estados do Maranhão, Pará, Rondônia e Tocantins.

\section{RESULTADOS E DISCUSSÃO}

Os resultados da análise documental da área de estudo encontraram 107 programas ambientais, destes o quantitativo e a distribuição seguem apresentados na (Tabela 1).

Tabela 1 - Total de Programas ambientais por área de estudo $(n=107)$

\begin{tabular}{lccc}
\hline \multicolumn{1}{c}{ Categoria } & $\mathbf{n}$ & $\%$ \\
\hline Área de estudo 1 & 52 & 48,60 \\
\hline Área de estudo 2 & 24 & 22,42 \\
\hline Área de estudo 3 & 31 & 28,98 \\
\hline Total de Programas ambientais & 107 & 100 \\
\hline
\end{tabular}

Fonte: Autores (2019).

A Área de estudo 1, UHE Belo Monte, apresentou 52 programas ambientais descritos nos estudos de impactos ambientais, destes, 4 programas e três projetos ambientais, são específicos à temática da saúde da população afetada.

A Área de estudo 2, UHE Santo Antônio, apresentou 24 programas ambientais descritos em seus estudos de impactos ambientais, destes um programa ambiental é específico à temática da saúde da população afetada.

Já a Área de estudo 3, UHE Estreito, apresentou 31 programas ambientais descritos nos estudos de impactos ambientais, destes, dois programas e dois subprogramas ambientais são específicos à temática da saúde da população afetada.

A designação específica descrita nos estudos de impactos ambientais de cada projeto hidrelétrico das áreas de estudo referentes à temática da saúde da população afetada é apontada na (Tabela 2).

Tabela 2 - Síntese dos Programas e Projetos Ambientais apontados no EIA das áreas de estudo

\begin{tabular}{|c|c|c|}
\hline Área de estudo & Hidrelétrica & Categorias (Programas e projetos ambientais de saúde) \\
\hline 1 & $\begin{array}{l}\text { UHE Belo } \\
\text { monte }\end{array}$ & $\begin{array}{l}\text { Programa de saúde e segurança, Projeto de controle médico, saúde } \\
\text { ocupacional e segurança do trabalho, Projeto de segurança e alerta, } \\
\text { Projeto de recomposição/adequação dos equipamentos e serviços de } \\
\text { saúde e Programa de incentivo à estruturação da atenção básica à } \\
\text { saúde, Programa de vigilância epidemiológica, prevenção e controle de } \\
\text { doenças e e Programa de Ação para } \\
\text { Controle da Malária (PACM). }\end{array}$ \\
\hline 2 & $\begin{array}{l}\text { UHE Santo } \\
\text { Antônio }\end{array}$ & $\begin{array}{l}\text { Programa de saúde pública, assistência à saúde da população, } \\
\text { Vigilância epidemiológica e vetorial, Monitoramento e controle de } \\
\text { vetores. }\end{array}$ \\
\hline 3 & UHE Estreito & $\begin{array}{l}\text { Programa de controle das condições de saúde, Sub-programa de } \\
\text { atendimento médico sanitário para a população residente na AID, } \\
\text { Sub-programa de atendimento médico sanitário para os trabalhadores } \\
\text { da obra e Programa de controle de vetores. }\end{array}$ \\
\hline
\end{tabular}

Total de Programas

e projetos (saúde)

10

Fonte: EIA, Belo Monte, Estreito e Santo Antônio. Elaborado pelos autores (2019). 
As três áreas de estudo $n=(107)$ apresentaram uma média de 35,66 de programas ambientais por projeto. Dentre elas, os programas ambientais específicos à temática da saúde da população afetada, apresentaram uma média de 2,33\%. Como se pode perceber, a categoria referente a área de estudo 1 apresenta um percentual menor de programas ambientais específicos à temática da saúde da população afetada em relação as demais áreas de estudo. A proporção média percentual de programas ambientais específicos à saúde da população afetada das três áreas de estudo soma 6,21\%.

A área de estudo 1 apresentou no seu contexto, programas ambientais específicos à temática da saúde da população afetada, abrangendo a saúde e segurança do trabalhador, estruturação da atenção básica, vigilância epidemiológica, prevenção e controle de doenças e controle da Malária em programa específico.

Comparando com as demais áreas de estudo, a área 1 apresenta maior abrangência em relação à saúde da população afetada. A área de estudo 2 apresenta no contexto de seus programas ambientais específicos à temática da saúde da população afetada, abrangência simplificada e consolidada em um único programa ambiental de saúde. Composto pela Assistência à saúde da população, vigilância epidemiológica e vetorial e monitoramento e controle de vetores.

A área de estudo 3 apresenta no contexto de seus programas ambientais específicos à temática da saúde da população afetada, abrangência dividida em: atendimento médico sanitário para a saúde da população e aos trabalhadores da obra. Traz um Programa específico para o controle de vetores.

É importante ressaltar que nas três áreas do estudo, empresas privadas que elaboram os estudos ambientais, bem como os programas ambientais. Não foram encontradas informações disponíveis sobre especialistas envolvidos na elaboração das categorias nas áreas 1 e 3. A metodologia de elaboração dos programas ambientais de saúde não foi descrita em nenhuma das áreas de estudo. No entanto, é possível apontar um padrão entre as mesmas referentes a temática estudada. Fato esse que se explica, por serem as mesmas empresas estarem elaborando essas categorias.

Não há informação sobre a participação das comunidades afetadas, bem como de seus representantes na elaboração dos programas ambientais. Para Gohn MG (2004) "a participação da sociedade civil" deve advir de estruturas que sejam participativas e organizadas de forma autônoma.

Percebe se a verticalização na elaboração dessas categorias. Para o governo brasileiro a população em geral obtem conhecimento dos estudos do projeto hidrelétrico pleiteado através do Relatório de Impacto Ambiental (RIMA), que é apresentado de forma objetiva, em linguagem acessível, ilustrado por mapas, cartas, quadros, gráficos e demais técnicas de comunicação visual, de modo que se possam entender as vantagens e desvantagens do projeto, bem como todas as consequências ambientais de sua implementação (BRASIL, 2015).

No entanto a publicidade dos estudos em questão, no caso do RIMA é uma exigência prevista na Constituição Brasileira, № art. 225. E ainda de acordo com Resolução CONAMA 009/87, o cidadão pode ter acesso para conhecimento e manifestação ao RIMA dos projetos de seu interesse, desde que reúna um grupo de no mínimo 50 outros interessados (BRASIL,1987).

A população afetada nesse processo participa de forma passiva, na parte final do processo e o órgão licenciador só realizara audiência pública se julgar necessário. A participação das populações afetadas deveria relacionar se ao ambiente como condição da sustentabilidade e assimilar-se a diversos paradigmas teóricos para internalizar os custos ecológicos do crescimento econômico, a eficácia energética dos processos produtivos, a racionalidade ecológica e valores conservacionistas do processo humano (LEFF E, 2000).

Percebe se quanto a forma de gestão, que os programas ambientais de saúde referentes a população afetada foram geridos e executados pelos empreendedores dos mesmos projetos hidrelétricos em realizam 0 estudo ambiental. Quanto ao monitoramento desses programas, apenas a área de estudo 3 apresentou nos estudos ambientais a previsão de forma específica ao programa ambiental de saúde. Para Sanches $\mathrm{R}$ (2011) o monitoramento fornece as condições básicas para avaliar a efetividade das medidas mitigadoras validando técnicas de previsão de impactos utilizadas. 
As 11 categorias de análise no contexto dos estudos ambientais na área de estudo, e apontam ainda onde o padrão desses projetos hidrelétricos diferenciou se (Figura 2).

Figura 2 - Categorias analisadas nos estudos de impactos ambientais (EIA) das três áreas de estudo.
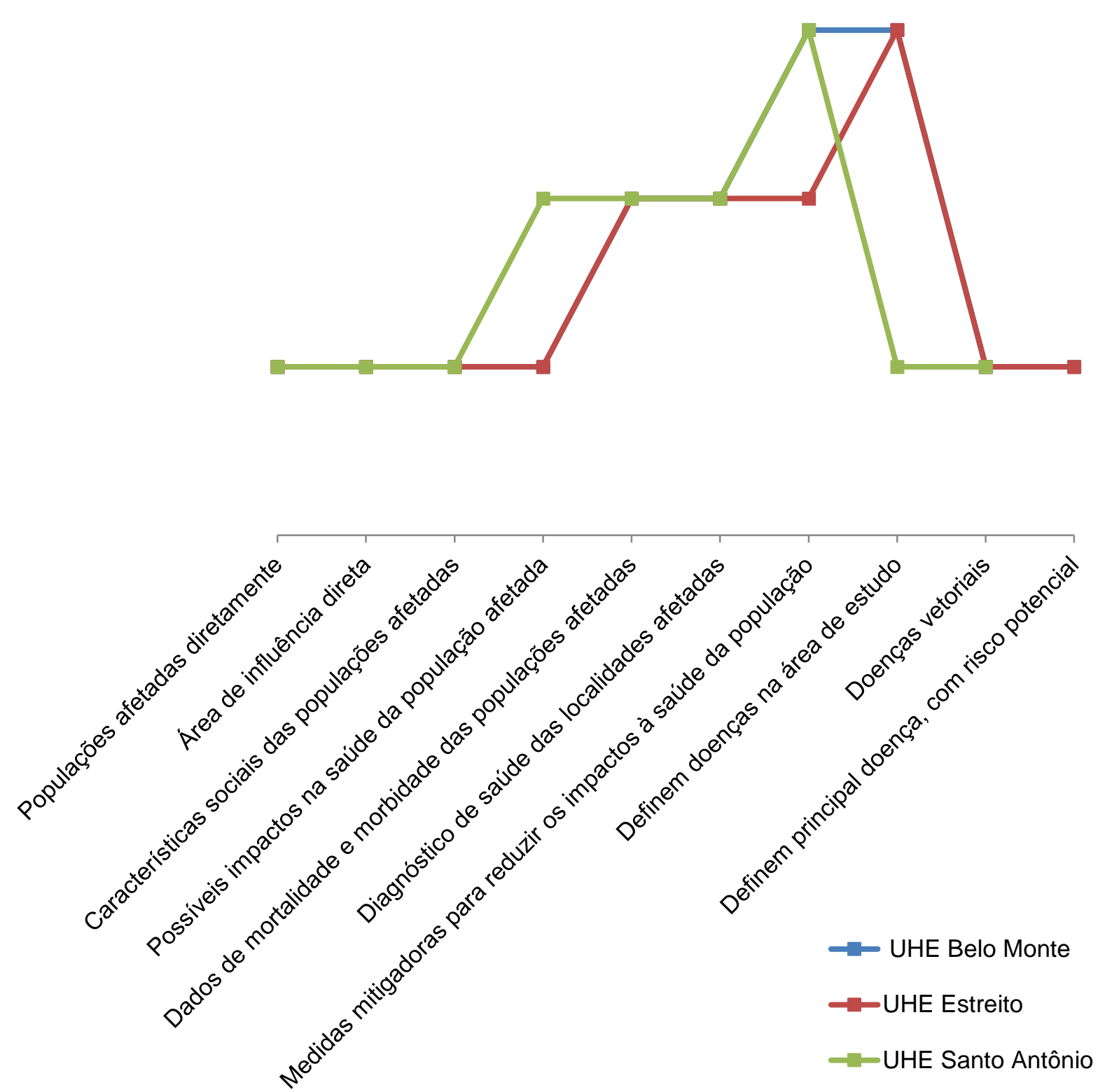

Fonte: Autores (2019).

As três áreas de estudo apontam categorias referentes as populações afetadas diretamente, definem a área de influência direta e apresentam características sociais das populações afetadas, também apontam a possibilidade de consequências na saúde da população afetada, gerada através dos impactos sobre o meio ambiente.

Quanto a mortalidade e morbidade das populações afetadas nas áreas de estudo, não indicam dados sobre essas categorias. Não indicam se houve diagnóstico de saúde das localidades afetadas para compor o embasamento dos programas ambientais de saúde. 
A categoria destacada como medidas mitigadoras para reduzir os impactos à saúde da população, é apontada parcialmente pelos planos, programas e projetos governamentais relacionados à saúde da população afetada na área de influência do projeto da área de estudo 1.

Ao ser comparada com as demais áreas de estudo apresenta se com padrão diferenciado nesse quesito. No EIA referente as áreas de estudo 2 e 3 não foram identificadas medidas mitigadoras específicas referentes a saúde da população afetada.

As três áreas de estudo definem de forma parcial as doenças relativas à saúde da população afetada, o foco principal são as doenças vetoriais, onde a Malária ocupa o foco central destacada como agravo de maior importância epidemiológica para a saúde da população afetada. A atenção à saúde da população afetada no contexto do licenciamento ambiental brasileiro em empreendimentos hidrelétricos estão inseridas de forma pulverizada nos programas ambientais de saúde. A maior parte da contemplação desses programas ambientais de saúde das áreas de estudo, ocorreu na atenção básica e vigilância em saúde. As populações afetadas não são oportunizadas a participarem do processo de elaboração dos programas ambientais de saúde que elas mesmas fariam jus a posteriori. Que a voz da população afetada pelos projetos hidrelétricos, seja ouvida de forma legitimada do início ao fim de sua concepção.

\section{CONSIDERAÇÕES FINAIS}

Evidencia-se a necessidade de se efetuar um balanço crítico do modelo que vem sendo usado para a construção dos programas ambientais de saúde. Medidas técnicas, científicas e epidemiológicas efetivas devem ser introduzidas, além das considerações ambientais no processo decisório do licenciamento ambiental.

\section{REFERÊNCIAS}

1. ANDRADE SM. O patrimônio histórico arqueológico de Serra da Mesa: A construção de uma nova paisagem. Tese (Doutorado em Geografia) - Pós-Graduação do Departamento de Geografia da Faculdade de Filosofia, Letras e Ciências Humanas. Universidade de São Paulo. São Paulo, 2002; 266p.

2. ANTUNES MM. Técnica Delphi: metodologia para pesquisas em educação no Brasil. Revista educação PUCCampinas, Campinas, 2014; 19(1): 63-7.

3. AYRES JM. As matas de várzea do Mamirauá: Médio Solimões. Cnpq. Sociedade civil Mamirauá. Brasília-DF, $2^{\circ}$ ed. $1995 ; 123 \mathrm{p}$.

4. BARBOSA EM, BARATA MM, HACON SD. A saúde no licenciamento ambiental: uma proposta metodológica para a avaliação dos impactos da indústria de petróleo e gás. Ciência e Saúde Coletiva. 2012; 17: 299-310.

5. BRASIL. Ministério da Saúde. Portaria ํo 104, de 25 de janeiro de 2011.

6. BRASIL. Conselho Nacional do Meio Ambiente. Resolução oㅜ 001, de 23 de janeiro de 1986. Publicada no D.O.U., de 17 de fevereiro de 1986; 2548-2549 p.

7. CANCIO J A. Inserção das questões de saúde no estudo do impacto ambiental. Dissertação (Mestrado em Planejamento e Gestão Ambiental)-Universidade Católica de Brasília, Brasília, 2008;95p.

8. CNEC, Engenharia S. A. Estudo de Impacto Ambiental e Relatório de Impacto Ambiental. Usina Hidrelétrica de Estreito. CNEC Engenharia S. A. São Paulo, SP, 2005.

9. EIA. Estudo de Impacto Ambiental UHE Belo Monte. Planos, Programas e Projetos Ambientais. Capítulo 12. 2009 ; $439 \mathrm{p}$.

10. EIA. Estudo de Impacto Ambiental UHE Estreito. Proposição de Programas Ambientais; 10.1. Estruturação dos programas. Capítulo 10. 2001; 309 p.

11. EIA. Estudo de Impacto Ambiental UHE Estreito. Análise integrada, avaliação de impactos, prognóstico e Programas Ambientais. Tomo C. CAPÍTULO 3. 2006; 321 p.

12. GOHN MG. Empoderamento e participação da comunidade em políticas sociais. Saúde e Sociedade, v. 13, 2004; 20-31.

13. HORBE AMC, et al. Geoquímica das águas do médio e baixo rio Madeira e seus principais tributários. Amazonas. Brasil. Revista Acta Amazônica, 2013.

14. IBGE. Instituto Brasileiro de Geografia e Estatística. Cidades, 2016. 
15. LEFF E. Saber Ambiental. Sustentabilidade, Racionalidade, Complexidade, Poder. Petrópolis. Vozes/PNUMA, 2001; $343 \mathrm{p}$.

16. MINAYO MCS. O desafio do conhecimento: pesquisa qualitativa em saúde. 8. ed. São Paulo, 2004; 269 p.

17. MIRANDA EEF. Efeitos ecológicos das barragens do Xingu: uma avaliação preliminar. Comissão Pró-Índio. São Paulo, 1988; $196 \mathrm{p}$.

18. NERES JCl. Avaliação ambiental da ocupação espacial do vale do Rio Tocantins por usinas hidrelétricas. Dissertação (Mestrado em Ciências Ambientais e Saúde)- Pró-Reitoria de Pós-Graduação e Pesquisa da Universidade Católica de Goiás. Goiânia, 2008.

19. SALOMÃO, et al. As florestas de Belo Monte na grande curva do rio Xingu, Amazônia Oriental. Boletim do Museu Paraense Emílio Goeldi Ciências Naturais, v. 2, n. 3, 2007; 55-153.

20. SANCHES RA. Avaliação de impacto ambiental e as normas de gestão ambiental da série ISO 14000 : características técnicas, comparações e subsídios à integração. Dissertação (Mestrado em Ciências da Engenharia Ambiental) - Escola de Engenharia de São Carlos, University of São Paulo, São Carlos, 2011. doi: 10.11606/D.18.2011.tde-25042011-103032.

21. SILVA J M, et al. Saúde: um tema ausente do licenciamento ambiental. Saúde do trabalhador e sustentabilidade do desenvolvimento humano-ensaios em Pernambuco. Recife: Editora Universitária da UFPE, 2009; 313-329.

22. SILVEIRA M. A implantação de hidrelétricas na Amazônia brasileira, impactos socioambientais e à saúde com as transformações no território: o caso da UHE de Belo Monte. Tese (Doutorado em Geografia) - Universidade de Brasília, 2016; 211 p. 